Emerson and the Climates of History 



\title{
Emerson and the Climates of History
}

\author{
\& Eduardo Cadava
}

Stanford University Press, Stanford, California 1997 
Stanford University Press, Stanford, California

(C) 1997 by the Board of Trustees of

the Leland Stanford Junior University

Printed in the United States of America

CIP data appear at the end of the book

Stanford University Press publications are distributed exclusively by Stanford University Press within the United States, Canada, Mexico, and Central America; they are distributed exclusively by Cambridge University Press

throughout the rest of the world. 
For my mother and father 
